Mass Profiles and Shapes of Cosmological Structures

G. Mamon, F. Combes, C. Deffayet, B. Fort (eds)

EAS Publications Series, Vol. ?, 2005

\title{
VELOCITY MOMENTS OF DARK MATTER HALOES
}

\author{
Wojtak, R. ${ }^{1}$, Łokas, E. ${ }^{2}$, Gottlöber, S. ${ }^{3}$ and Mamon, G. ${ }^{4}$
}

Abstract. Using cosmological N-body simulations we study the line-ofsight velocity distribution of dark matter haloes focusing on the lowestorder even moments, dispersion and kurtosis, and their application to estimate the mass profiles of cosmological structures. For each of the ten massive haloes selected from the simulation box we determine the virial mass, concentration and the anisotropy parameter. In order to emulate observations from each halo we choose randomly 300 particles and project their velocities and positions along the line of sight and on the surface of the sky, respectively. After removing interlopers we calculate the profiles of the line-of-sight velocity moments and fit them with the solutions of the Jeans equations. The estimates of virial mass, concentration parameter and velocity anisotropy obtained in this way are in good agreement with the values found from the full 3D analysis.

The purpose of this study was to verify how well the global properties of dark matter haloes can be reproduced from the joint fitting of the line-of-sight velocity dispersion and kurtosis profiles to the solutions of the Jeans equations, a method first applied by Łokas \& Mamon (2003) to the Coma cluster. Our approach is similar to that of Sanchis et al. (2004). We have used ten massive haloes (with masses larger than $10^{14} \mathrm{M}_{\odot}$ ) extracted from a cosmological simulation of the ' standard $\Lambda$ CDM model performed in a box of size $150 \mathrm{Mpc} / h$ (Wojtak et al. 2005). We determined the properties of the haloes estimating their virial mass, concentration (as defined in the NFW formula) and anisotropy parameter.

To mimic observations we project velocities and positions of the halo particles along the line of sight of an imaginary observer placed at the distance of $100 \mathrm{Mpc}$ from a halo and onto the plane of the sky respectively, producing velocity diagrams $V(R)$. For each halo we choose randomly 300 particles with velocities in the

\footnotetext{
${ }^{1}$ Astronomical Observatory of Jagiellonian University, Cracow, Poland

2 Nicolaus Copernicus Astronomical Center, Warsaw, Poland

3 Astrophysikalisches Institut Potsdam, Germany

${ }^{4}$ Institut d'Astrophysique de Paris, France
} 

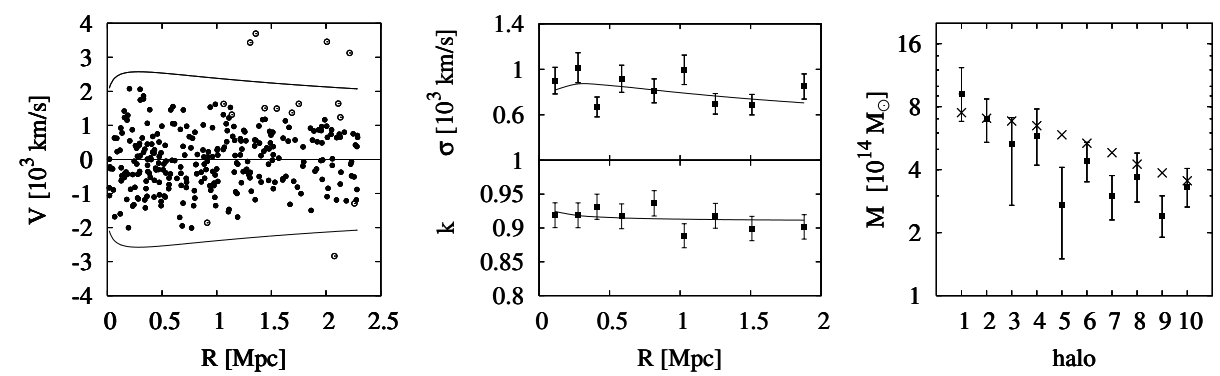

Fig. 1. Left panel: the velocity diagram with filled (empty) circles denoting bound (unbound) particles and curves separating interlopers from members. Middle panel: velocity dispersion and kurtosis variable $k$ with curves showing the fitted solutions of the Jeans equations. Right panel: virial mass estimates for ten haloes obtained in 3D analysis (crosses) and in the Jeans analysis (squares).

range $+/-4000 \mathrm{~km} / \mathrm{s}$ with respect to the halo mean velocity. In order to remove interlopers from our samples for each halo we compute the line-of-sight velocity dispersion profile $\sigma(R)$ and fit it with solutions of the Jeans equation assuming isotropic orbits. We reject as interlopers the particles with velocities outside the fitted range $+/-3 \sigma(R)$ with respect to the halo mean velocity, repeating the procedure until convergence is achieved. An example of the velocity diagram for the most massive halo in our sample is shown in the left panel of Fig 1 together with the results of this procedure.

After removing interlopers we measure final profiles of the line-of-sight velocity moments: dispersion and kurtosis. Then we fit these data with solutions of the Jeans equations (Lokas \& Mamon 2003) obtaining estimates of virial mass, concentration and anisotropy parameter. The middle panel of Fig 1 shows the velocity dispersion and kurtosis variable $k$ (related to the estimator of kurtosis $K$ by $\left.k=[\log (3 K / 2.68)]^{1 / 10}\right)$ for our most massive halo.

The fitted parameters are in good agreement with values obtained from the full 3D information. As an example we show in the right panel of Fig 1 values of the virial mass for the whole sample of ten haloes found in the full 3D analysis (crosses) and in the Jeans analysis (squares with errors following from the sampling errors of velocity moments). We conclude that the method of joint fitting of line-of-sight velocity dispersion and kurtosis reproduces basic parameters of dark matter haloes in a satisfactory way.

\section{References}

Łokas, E., \& Mamon, G., 2003, MNRAS, 343, 401

Sanchis, T., Łokas, E., \& Mamon, G., 2004, MNRAS, 347, 1198

Wojtak, R., Łokas, E., Gottlöber, S., \& Mamon, G., 2005, MNRAS, 361, L1 